\title{
EDITORIAL
}

\section{Connecting the dots: obesity, fatty acids and cancer}

\author{
Philip A Wood
}

Obesity is becoming recognized as a common co-morbidity with several disease states including cancer. There is tremendous interest to more fully understand the mechanistic connections between obesity and cancer. This commentary highlights the report by Byon et al in this issue of Laboratory Investigation. It provides an interpretation of their results in the context of other disease states involving obesity and its sequelae of excess fatty acids and increased plasminogen activator inhibitor-1 (PAl-1) concentrations. This includes obesity-related conditions, in particular metabolic syndrome, and its subphenotypes such as insulin resistance, and increased risk of myocardial infarction. The potential mechanistic tie-ins of obesity, elevated concentrations of fatty acids, as well as elevated PAI-1 levels with cancer and its risk for aggressiveness are discussed.

Laboratory Investigation (2009) 89, 1192-1194; doi:10.1038/labinvest.2009.99

T here is a great deal of interest in the observation that certain types of cancer are often associated with obesity. There are accumulating data that support the notion that cancer should be added to the increasing list of obesity-related diseases or disease processes such as nonalcoholic fatty liver disease, insulin resistance, type II diabetes and cardiovascular disease. Cancers associated with obesity include some of the most prevalent types including colon, breast, prostate and endometrial. ${ }^{1}$ Is there a 'cause and effect' relationship with the obese state, a condition of excess fatty acids, and increasing the risk of cancer or increasing the virulence of cancer, that is, being more invasive and metastatic? Or do they happen to occur together as independent events?

Obesity is well recognized as a risk factor for developing type II diabetes. This process is often associated with excessive adipose tissue, especially visceral adiposity, which provides a continual source of excess fatty acids for the body to respond to, particularly the liver. Could it be that the excess fatty acid environment of obesity likewise has a direct role in promoting the development or modifying the aggressiveness of cancer? Thus, there are many shared factors and biomarkers of interest between patients with obesity, insulin resistance, metabolic syndrome, type II diabetes and those with cancer. ${ }^{2}$ Plasminogen activator inhibitor-1 (PAI-1) is one such potential marker. $^{1,3}$

As reported in this issue of Laboratory Investigation, Byon et al have shown a potential chain of events directly linking fatty acidstimulated PAI-1 expression via SMAD-4 activation that was associated with aggressiveness (migration) of breast cancer cell lines in culture. SMAD4 was stimulated by excessive fatty acids in the medium, notably the ubiquitous dietary omega- 6 fatty acid, linoleic acid, as well as oleic acid to a lesser extent. Linoleic acid is a common omega- 6 fatty acid found in the Western diet especially in foods prepared from plant-derived polyunsaturated fats, for example, safflower oil is an excellent source. It is of interest that a recent American Heart Association Panel reports ${ }^{4}$ that at least $5-10 \%$ of energy derived from omega- 6 fatty acids reduces the risk of cardiovascular disease relative to lower intakes. Thus, it remains to be seen if this recent recommendation affects cancer risks. Oleic acid, another common dietary fatty acid found enriched in olive oil and canola oil is likewise of interest because it is often associated with more healthful properties regarding raising HDL-cholesterol and lowering LDL-cholesterol, ${ }^{5}$ thus it may help improve the dyslipidemia found commonly in obesity. 
PAI-1 is also elevated in obesity-related conditions, and is considered a common risk factor for myocardial infarction associated with metabolic syndrome. ${ }^{3}$ Increased PAI- 1 concentrations are a potential contributor in the pro-thrombotic state found in these patients, ${ }^{3,6}$ as well as a prognostic biomarker for breast cancer as described in Byon et al in this issue of Laboratory Investigation. Although further clarification of the mechanisms remains to be defined, Byon, et $a l^{1}$ essentially fulfill a 'Koch's Postulates' like score card in their experiments. They showed that MDA-MB 231 breast cells are stimulated to produce more secreted PAI-1 via fatty acid (linoleic acid, and to a lesser extent oleic acid) stimulation, which was clearly associated with increased aggressiveness as measured by the migration assay. Other fatty acids with different chain lengths and saturation states had no effect as far as PAI-1 expression or cell migration. Furthermore, the linoleic acid migration was inhibited by expression of antisense RNA against PAI-1. SMAD4 appeared to be the mediator of this fatty acid-stimulated event, and that was further elucidated by showing that SMAD4deficient MD-MB-468 breast carcinoma cells lacked the response. The translation of the mechanism reported by Byon et al ${ }^{1}$ is speculated in Figure 1.

In my mind these studies raise a number of interesting issues regarding fatty acids, PAI-1 concentrations and cancer. Are there additional mechanisms involving fatty acid metabolism that would affect cancer cell behavior? For example, there is a lot of interest in the role of excess fatty acids in the development of insulin resistance in normal cells such as hepatocytes or muscle cells. There are several mechanistic hypotheses ranging from deficient mitochondrial fatty acid oxidation with a build-up of fatty acid-related metabolites such as diacylglycerols and activation of PKC $\varepsilon / \theta$ resulting in decreased insulin signaling ${ }^{7}$ to other mechanisms that propose excessive, but incomplete mitochondrial fatty acid oxidation resulting in mitochondrial stress and insulin resistance. ${ }^{8}$ Other ideas are that elevated fatty acids in the circulation leads to increased rates of both cellular fatty acid transport and mitochondrial oxidation at the expense of glucose oxidation resulting in a back up of glucose consumption by those cells, essentially appearing as insulin resistance, ${ }^{9}$ but without direct connection to insulin signaling per se. Cancer cells are notorious for their metabolism based on glucose utilization via aerobic glycolysis ${ }^{10}$ or what is known as the 'Warburg Effect,' and this process could be affected by fatty acid effects on cellular uptake and consumption of glucose. Furthermore, since hyperinsulinemia is extremely common in obesity, what would be the metabolic effects on cancer cells bathed in high insulin concentrations? There are recent epidemiological studies indicating an association ${ }^{11}$ between elevated insulin and risk of breast cancer. Additional mechanisms to consider between fatty acid metabolism and cancer include the potential generation of reactive oxygen species as a result of abundant polyunsaturated fatty acids such as linoleic acid that may be chemically oxidized in the oxidative stress conditions of obesity. ${ }^{2}$ Furthermore, the cyclooxygenase-2 (COX-2) is a key step in the prostanoid biosynthetic pathway. COX-2 uses as substrate arachodonic acid, which is downstream from linoleic acid, and appears to have an important role in the carcinogenesis of colorectal cancer. ${ }^{12}$ Finally, the crucial enzyme of fatty acid synthesis, fatty acid synthase (FAS) is associated with a poor prognosis in breast and prostate cancers as FAS is abundant in many human carcinomas. $^{13}$

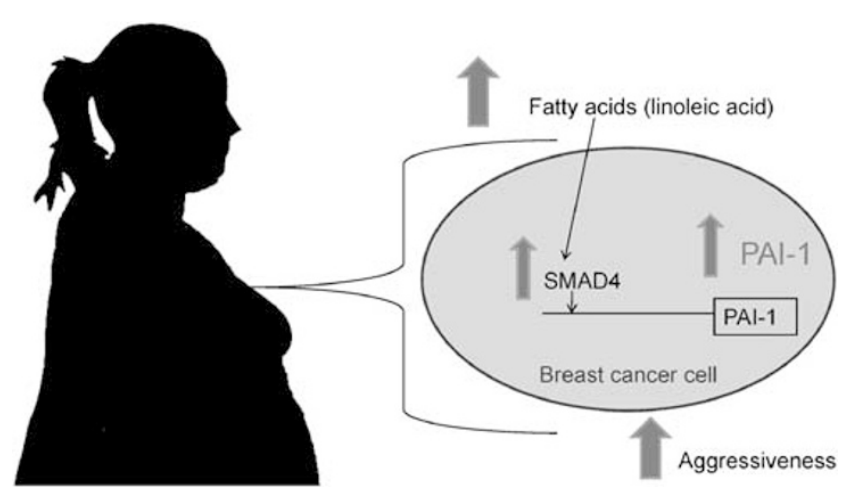

Figure 1 Summary mechanism relating increased fatty acid stimulation of PAl-1 expression in the context of obesity and breast cancer. 
In summary, the cell culture study reported by Byon et $a^{1}$ extends our understanding of potential mechanisms beyond the previous observations of the association between obesity and cancer in which excessive fatty acids, especially linoleic acid, may be a metabolic link. In addition, they have helped further define the potential value of PAI-1 as a risk factor based on the mechanistic studies reported. Further data to support informative biomarkers useful as risk factors will help better define cancers that can be expected to respond well to conventional therapies and those that will be untreatable. Further in vivo studies, however, will be important to verify and extend the results reported by Byon et $\mathrm{ll}^{1}$. It is striking that obesityrelated phenotypes such as metabolic syndrome share features with cancer prevalence and specifically in this study this includes increased concentrations of fatty acids and PAI-1. The downside of course is that these similarities make sorting out 'cause and effect' more difficult.

\section{DISCLOSURE/CONFLICT OF INTEREST}

The author declares no conflict of interest

1. Byon $\mathrm{CH}$, Hardy RW, Ren $\mathrm{C}$, et al. Free fatty acids enhance breast cancer cell migration through plasminogen activator inhibitor-1 and SMAD4. Lab Invest 2009;89: 1121-1228 (this issue).

2. Cowey S, Hardy RW. The metabolic syndrome: a high-risk state for cancer? Am J Pathol 2006;169:1505-1506.
3. Mertens I, Verrijken A, Michiels JJ, et al. Among inflammation and coagulation markers, PAl1 is a true component of the metabolic syndrome. Int J Obesity 2006;30:1308-1314.

4. Harris WS, Mozaffarian D, Rimm E, et al. Omega-6 fatty acids and risk for cardiovascular disease. Circulation 2009;119:902-907.

5. Hu FB, Manson JE, Willett WC. Types of dietary fat and risk of coronary heart disease: a critical review. J Am Coll Nutr 2001;20:5-19.

6. Boronat $M$, Saavedra $P$, Varillas VF, et al. Use of confirmatory factor analysis for the identification of new components of the metabolic syndrome: The role of plasminogen activator inhibitor-1 and Haemoglobin A1c. Nutr Metab Cardiovasc Dis 2009;19:271-276.

7. Savage DB, Petersen KF, Shulman Gl. Disordered lipid metabolism and the pathogenesis of insulin resistance. Physiol Rev 2007;87:507-520.

8. Koves TR, Ussher JR, Noland RC, et al. Mitochondrial overload and incomplete fatty acid oxidation contribute to skeletal muscle insulin resistance. Cell Metab 2008;7:45-56.

9. Finck BN, Bernai-Mizrachi C, Ho-Han D, et al. A potential link between muscle peroxisome proliferators-activated receptor- $\alpha$ signaling and obesity-related diabetes. Cell Metab 2005;1:133-144.

10. Vander-Heiden MG, Cantley LC, Thompson CB. Understanding the Warburg effect: the metabolic requirements of cell proliferation. Science 2009;324: 1029-1033.

11. Kabat GC, Kim M, Caan BJ, et al. Repeated measures of serum glucose and insulin in relation to postmenopausal breast cancer. Int J Cancer 2009 (in press).

12. Brown JR, DuBois RN. COX-2: A molecular target for colorectal cancer prevention. J Clin Oncol 2005;23: 2840-2855.

13. Kuhajda FP. Fatty acid synthase and cancer: new application of an old pathway. Cancer Res 2006;66: 5977-5980. 